\title{
Keyword index to volume 8
}

\author{
1 p36 deletion syndrome 764 \\ $22 q 11801$ \\ 35delG 19 \\ 3D model 280 \\ 657del5 900 \\ $7 q 11.23$ deletion 215 \\ $7 q 35174$ \\ $8 q 13319$ \\ $\alpha$-foetoprotein 986 \\ Aarskog syndrome 869 \\ accuracy 181 \\ ACE 545 \\ admixture 119 \\ admixture test 399 \\ adrenal hypoplasia 87 \\ adult vitelliform macular dystrophy 286 \\ aetiology 267 \\ agangliosis 393 \\ age of onset 145 \\ age-related macular degeneration 286 \\ airway responsiveness 853 \\ Albania 480 \\ Albright-like syndrome 597 \\ Alzheimer's disease 259, 259, 713 \\ aminoglycoside 315 \\ AMOVA 480 \\ ancient DNA 669 \\ anticipation 4 \\ APC germline mutation 42 \\ apolipoprotein B 621 \\ ARPKD 163 \\ array-bound single nucleotide primer exten- \\ sion 884 \\ arteries 704 \\ aspartoacylase 557 \\ asphyxiating thoracic dystrophy 645 \\ association 788 \\ association analysis 473 \\ association studies 154 \\ association study 721 \\ assortative mating 119 \\ asthma 788,853 \\ ataxia 986 \\ autosomal dominant 443
}

autosomal dominant cerebellar ataxia 4

autosomal dominant congenital cataract 535

autosomal dominant type lla hypercholester-

olaemia 621

autozygosity mapping 991

Axenfeld-Rieger anomaly 71

Axenfeld-Rieger syndrome 71

baculovirus 95

Bac/Yac contig 527

Becker muscular dystrophy 87, 293

Best disease 286

bestrophin 286

BFNC 994

bHLHZip domain 215

biobank 739

biparental 641

bipolar disorder 385

blood eosinophil count 853

BMD 793 branch-point 311

BRCA1 130, 204

BRCA2 130, 204

breast cancer 204, 731, 757

breech presentation 571

Busselton Population Health Study 853

CADASIL 280

CAG repeat disorder 631

CAG/CTG 385

Canavan disease 557

cancer 540

carrier detection 293

carrier frequency 19, 367, 900

carrier rate 307

carrier testing 79

cat eye syndrome 801

cathepsin K ( 431

cationic trypsinogen gene 473

CDKN2A 590

CEPH families 33

cerebellar 986

cerebral palsy 267

Charcot-Marie-Tooth 229

chemokine receptors 975

chromosomal mapping 923

chromosomal translocation 875

chromosome 12613

chromosome 12p11.2 561

chromosome 18385

chromosome 19809

chromosome 20535

chromosome 22895

chromosome 2q 597, 991

chromosome 4p16 645

chromosome 6 uniparental disomy 137

chromosome 6p25 71

chromosome 8875

chromosome 8p21 273

chromosome aberrations 661

chromosome analysis 661

chromosome evolution 167

Chromosome locus 18p11.32-p11.23 443

chromosome region (2)(q21q23) 393

chronic granulomatous disease 697

Chuetas 242

clinical and genetic correlations 4

clinical management 204

clinical manifestation 519

CMT1A 229

cognitive impairment 552

comparative genomic hybridisation 661

comparative mapping 167

complex disease 154, 399

complicated spastic paraplegia 777

congenital adrenal hyperplasia 827

congenital alopecia 273

Connexin 141, 141

contraction 423

control region 964

coronary artery disease 389

craniosynostosis 571

CTG repeat 423

cultural inheritance 980

cyclopia 797 cystic fibrosis S2, 717

cystic fibrosis transmembrane conductance

regulator ( 473

cystogenesis 487

database 739

deafness 141, 141, 437

deletion 449, 597, 801

deletion size 519

deletion/duplication 63

demography 980

denaturing gradient gel electrophoresis 49 denaturing high-performance liquid chroma-

tography 24

developmental abnormalities 137

DFNA16 991

DFNB27 991

DFNB9 437

DGGE 353

diabetes 195

diagnostic testing 293

diaphyseal aclasis 24

DiGeorge syndrome 801

disease 195

distal monosomy $13 q 637$

distal trisomy 8q 637

DMPK 507

DNA bank 653

DNA extraction 653

DNA pools 933

dominant negative effect 771

dosage compensation 103

Down syndrome 223

Dp71 552

Duane syndrome 319

Duchenne muscular dystrophy 293, 552

duplication 449

dwarfism 645

dynein 923

dyschromosomal 661

dysfunction of the phototransduction cas-

cade 578

dystonia 464

dystrophin 87

dystrophin isoforms 552

EBV 460

EDM 125

Edwards syndrome 223

EGF-like repeat 280

elastin 955

Ellis-van Creveld 645

encryption 739

epilepsy 946

ERS 793

estimation 980

ethics 739

etiology 315

evaluation 204

evolution 209, 827, 923

exon-intron structure 381

exon skipping 311,793

expanded populations 604 
familial 641

familial adenomatous polyposis 731

familial defective apolipoprotein B-100 621

familial hypercholesterolaemia 621

family cancer history 181

Fanconi anaemia 861

FAP 42

fatty-aldehyde dehydrogenase ( 299

Fechtner syndrome 895

FGD1 869

FGDY 869

filensin 535

Finnish population 604

FISH 167, 293, 455, 764, 801

FKHL7 71

fluorescence 407

fluorescent ddNTPs 884

FMF 242, 307

foetal head constraint 571

foetal wastage 367

forkhead-like 771

founder effect $372,431,631,783,918$

founder mutation 861

founder mutations 757

Fragile X 247

FRAXE 157, 583

French Canadian population 372

French population 33

gain of function 507

gap junction 141,141

Gaucher 95

gene conversion 473

gene flow 750

gene mapping 407, 545

genealogy 743

genetic counselling 130, 157, 229

genetic deafness 19

genetic diversity 669

genetic epidemiology 315

genetic heterogeneity $4,473,578,621$

genetic homogeneity 372

genetic linkage 273

genetic mapping 372

genetic predisposition 473

genetic testing S2, 731

genetics $181,267,641,739,743,783,853$

946

genome scan 145

genomic deletion 259, 259

genomic structure $174,493,725$

genotype-phenotype correlations 42,157 ,

$578,683,717$

genotyping methods 154

germline 423

germline mosaicism 820

germline mutations 590

giant axon 527

Gilles de la Tourette syndrome 875

GJB2 19

global populations 975

glycerol kinase deficiency 87

GSTP1 540

guidelines S2, 204

haemochromatosis 389

hairless gene 273

haploinsufficiency 507,771

haplotype $431,583,678,757$

haplotype analysis $527,535,918$ hearing impairment 500

hearingimpairment 464

heavy chain 923

hemizygosity 215

hereditary breast and ovarian cancer 130

hereditary hearing loss 315

hereditary hypotrichosis simplex 443

hereditary multiple exostoses 24

hereditary non-polyposis colorectal cancer 49

hereditary pancreatitis (HP) 473

heteroduplex analysis 49

heterogeneity 109, 649

heterozygote advantage 367

hidrotic ectodermal dysplasia 372

Hirschsprung disease 721

HIV-1 975

HLA 119, 460

HLA haplotypes 33

HNPCC 347

Hodgkin's disease 460

holoprosencephaly 797

homologous recombination 63

homozygosity 986

homozygosity mapping 267, 527, 613, 991

human 407, 923

human 381

human genetic diversity 480

human longevity 743

human populations 750

Hungary 339

Huntington's disease 145, 678, 731

Hutterites 119

hybrid genes 827

hydatidiform mole 641

hydroxylase 683

hypercalciuria 414

hyperphenylalaninaemia 683

hypomagnesaemia 414

IBD1 846

Iceland 743

idiopathic generalised epilepsy 994

idiopathic mental retardation 637

IgE 853

immotile cilia syndrome 109

immunoglobulin 986

imprinting 137

inbreeding 119, 613

incomplete penetrance 704

Indian populations 678

Inflammatory bowel disease 846

inheritance 743

insertion 697

instability 423, 540

Interleukin-1 receptor 87

interleukin 9 receptor 788

interphase FISH 223

interstitial deletions 764

intra-uterine growth 137

intrachromosomal insertion 393

intrauterine environment 571

inv dup(22) 801

inverted duplications 597

inverted duplicons 597

iron 389

isochromosome 709

isolated hair abnormality 443

isolated populations 604

isolation by distance 750
Israel 307

Israeli Jewish melanoma families 590

Jews 242, 307

Kartagener syndrome 109

Kennedy disease 631

keratoderma 141, 141

kidney cysts 487

laboratory validation 293

Lafora 946

large pedigrees 545

LDL receptor 621

Leber's congenital amaurosis 578

Léri-Weill syndrome 54

limb development 561

LINE-1 697

linguistic boundaries 750

linguistics 480

linkage 125, 145, 389, 460, 649, 809, 846

linkage analysis 109, 473, 535, 777, 895

linkage disequilibrium 33, 527

Linkage disequilibrium 545

linkage disequilibrium 604, 613, 713, 918, 980

linkage disequilibrium analysis 372, 399

linkage heterogeneity 399

locus heterogeneity 991

LOH 487

lung function 853

lymphocyte 347

Madelung deformity 54

malignant hyperthermia 149

malignant myeloid disorders 174

mapping 535, 986

Marie-Unna hypotrichosis 273

May-Hegglin syndrome 895

MECP2 325

MEFV 242

meiotic errors 911

MELAS 315

meningococcal disease 513

mental retardation $87,157,253$

methylenetetrahydrofolate reductase 725

MHC 33

microdeletion 455,805

microdissection 393

microsatellite instability 939

microsatellite markers 604, 809

microsatellites 360

minisequencing 884, 933

mismatch distribution 964

missense mutation 473

missplicing 697

mitochondria 195

mitochondrial diseases 331

mitochondrial DNA 480,669

mitochondrial dysfunction 464

mitochondrial encephalomyopathy 315

molecular analysis 493

molecular cloning 407

molecular diagnosis 837

molecular modeling 500

monosomy 9p 167

Monte Carlo simulations 750

mosaicism 223, 423, 709

mRNA decay 299

mtDNA 331, 964 
mtDNA repair system 331

mtDNA variations 339

MTHFR 725

multifactorial diseases 604

multiple deletions 331

multiplex FISH 909

mutation $71,141,141,195,280,353,431$,

$437,571,697,704,869,946$

mutation analysis 95, 187, 286, 493, 994

mutation detection $24,49,557,884$

mutation rate 964

mutation screening 299, 347, 861

mutation testing $\mathrm{S} 2$

mutational hotspot 820

mutations 149, 307, 389, 513, 827, 955

mutations in the retinal-specific guanylate

cyclase gene 578

myelin disorder 837

myoclonus 946

myopathy 125

myotonic dystrophy 507

$\mathrm{N}$-glycosylation 367

nail patella syndrome 311

NBS 900

neighbour joining tree 964

neolithic 669

neonatal diabetes mellitus 137

nephrocalcinosis 414

Netherlands 827

neurofibroma 939

neurofibromatosis type 1209

neuromuscular disease 493

neuronal ceroid lipofuscinosis 381

neuropathy 986

neuroprotective allele 95

non-homologous recombination 259, 259

nonsense mutation 793

North Africa 360, 613

novel method 103

oligogenic inheritance 399

ovarian cancer 731,757

P1 protein 163

p16 590

pairwise differences 964

paleolithic 669

Palóc 339

pancreatitis 473

PAR 1805

PAR2 805

paracellin-1 414

paracentric inversion 21q 815

parental origin 911

parental origin of deletion 519

Patau syndrome 223

PCR 229

Pelizaeus-Merzbacher disease 449, 837

Pendred syndrome 437

Pendrin 437

phenotype 95

phenotype/genotype correlation 187

phenylalanine 683

phenylketonuria 683

phosphomannomutase 367

physical location 493

physical mapping 319, 561

physician 204

phytanic acid 649
PITX2 71

PKD1 353

PKHD1 163

PLP 449

PMD 449

point mutation 315

point mutations 552

polyglutamine expansion 4

polymorphic markers 163

polymorphism 141, 141, 540, 946

population genetics $33,315,360$

Portugal 540

positional cloning 875

post-natal growth 137

posterior polar cataract 535

potassium channel 994

power 119

predictive testing 130,731

premature ovarian failure 247

premutation 247

prenatal diagnosis 229, 557

presenilin-1 259, 259

preserved speech variant 325

prevalence 315

primary ciliary dyskinesia 109, 923

privacy 739

progressive 946

progressive epilepsy with mental retardation 381

promoter 713,725

promoter methylation 939

properdin deficiency 513

propionic acidaemia 187

protein truncation test 49,299

proteolipid protein gene (PLP) 837

pseudoautosomal region 788

pseudogene 174, 209

psychological aspects 130

psychological consequences 731

PTPase 946

PTT 42, 793

public health 204

pulmonary diseases 717

pycnodysostosis 431

quality assurance $\mathrm{S} 2$

quantitative trait 545

Quebec 980

recessive 986

recessive mutation 367

recessive X-linked 704

recombination 229, 805, 980

recommendations S2

recurrent 641

Refsum's disease 649

reinversion 815

relative hazard 975

relative risk 743

renal-coloboma syndrome 820

renal failure 414

repeated sequences 63,331

resistant polymorphisms 975

retinal dystrophy 783

retinitis pigmentosa 500,783

Rett syndrome 325

reverse-transcriptase polymerase chain

reaction 299

ring chromosome 801

RNA splicing 721 robot 653

robustness 119

RT-PCR 103

ryanodine receptor 149

sample bank 739

sarcoidosis 717

SBMA 631

Scandinavia 918

Segmental neurofibromatosis 455

segregation analysis 846

sequence alignment 407

sequence analysis 209

serotonin transporter 75

sex-chromosome non-disjunction 805

short-rib polydactyly 645

short stature 54

SHOX 54

sib pairs 460

single nucleotide polymorphisms 154, 721 933

single strand conformation polymorphism 49

single-strand conformation polymorphism

analysis 24

situs inversus 109

Sjögren-Larsson syndrome 299

skewed X-inactivation 253

Slav populations 900

SMA 79

SMN interacting protein 1 (SIP1) 493

SNP 545

SNP detection 24

somatic mosaicism 455

spastic paraplegia 771

spastic paraplegia (SPG) 837

spastin 771

spectrum of mutations 500

SPG4 771

spinal muscular atrophy 79,493

spinocerebellar ataxia 4, 731, 918

splice mutation 771

splice site mutation 311

splicing variant 347

stability 583

steroid 21-hydroxylase deficiency 827

STR 540

STRs 360

Strumpell's disease 771

substitution E318G 259, 259

subtelomeric 764

subtelomeric probes 909

subtelomeric translocation 637

supravalvular aortic stenosis 955

survival motor neuron 79

survival motor neuron gene ( 493

synpolydactyly 561

systematic review 731

telomere capture 764

telomere healing 764

terminal deletions 764

third-party 739

transcription factor 215

transcriptional map 777

transcripts 725

translocation 709

translocation trisomy 21815

transposition 704

trinucleotide repeat 385, 507, 583, 631

Triple A syndrome 613 
triploidy 911

trisomy 223

trisomy 21709

Turner syndrome 54

two-hit 487

two-locus models 399

type 1 diabetes 145

Usher syndrome type II 500

vacuolar neuromyopathy 809

variance components 853

verification 181
VMD2 286

Vohwinkel's syndrome 141, 141

voltage sensor 994

Williams-Beuren syndrome 215

Wilson disease 933

Wolf-Hirschhorn syndrome 519

Wolfram syndrome 145

X chromosome 63, 125, 837

$X$ inactivation 103, 449

$X$-inactivation 513

X-ray crystal structure 683
XLMR carriers 253

XMEA 125

Xp22-21 87

Xq22 87

XY sex reversal 167

Y-chromosome 480

Y chromosome polymorphisms 339

YAC 167

YAC contig 75

YAC fragmentation 385 